Oper Orthop Traumatol 2013 $\cdot 25: 416$

DOI 10.1007/s00064-013-0266-z

Online publiziert: 27. September 2013

(c) Springer-Verlag Berlin Heidelberg 2013

\title{
D.C. Wirtz
}

Klinik und Poliklinik für Orthopädie und Unfallchirurgie, Zentrum für Physiotherapie, Universitätsklinikum Bonn

\section{Hüftdysplasie}

net. Das dargestellte Spektrum reicht von der Arthrographie des Hüftgelenks mit geschlossener Reposition beim Neugeborenen/Säugling (Placzek et al.) über die Dega-Acetabuloplastik beim Kleinkind (Mladenov et al.) und Salter-PembertonOsteotomie im Kindesalter (Jäger et al.) bis hin zur dreifachen Beckenosteotomie nach Tönnis im Jugend- und Erwachsenenalter (Zahedi et al.) und endoprothetischen Versorgung bei Dysplasiecoxarthrose (Kohlhof et al.).

Dabei sei an dieser Stelle ausdrücklich darauf hingewiesen, dass schon in früheren Heften von Operative Orthopädie und Traumatologie mehrere Beiträge zum Thema Hüftdysplasie erschienen sind (s. Literaturverzeichnis).

Zusammengefasst bieten all diese beschriebenen operativen Methoden ein vollumfängliches Portfolio zur Behandlung der Hüftgelenkdysplasie. Für jede Ausgangssituation kann das entsprechend indizierte Verfahren nachgelesen werden. Damit wird der wesentlichen Zielsetzung unser Zeitschrift Rechnung getragen, „über die Jahre hinweg“ sowohl etablierte evidenzbasierte als auch neue innovative Operationsverfahren „zusammenzutragen". Aus Sicht der Herausgeber ist dieses Konzept einer „Operationslehre“ weiterhin attraktiv, um auch in Zukunft einen Beitrag zur möglichst optimalen Versorgungsqualität unserer Patienten zu leisten.

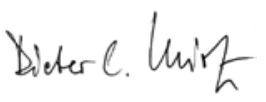

D.C. Wirtz

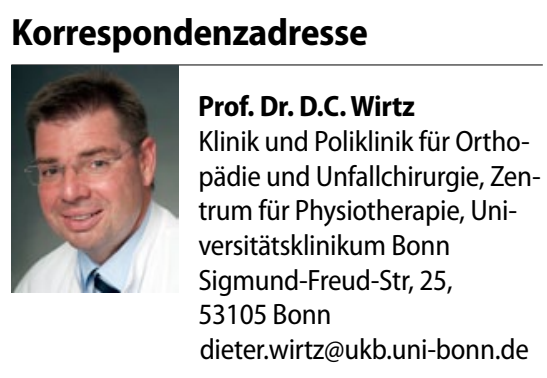

Einhaltung ethischer Richtlinien

Interessenkonflikt. D.C. Wirtz gibt an, dass kein Interessenkonflikt besteht.

\section{Literatur}

1. Küsswetter W, de Pellegrin M (1990) Die Saltersche Beckenosteotomie (Innominationsosteotomie) als hüftpfannenverbessernder Eingriff. Operat Orthop Traumatol 2:281-291

2. Carstens C, Schuknecht B (1996) Die offene Reposition des luxierten Hüftgelenkes im Kindesalter. Operat Orthop Traumatol 8:262-270

3. Brunner R, Baumann JU (2000) Die Rekonstruktion des luxierten Hüftgelenkes bei spastischer infantiler Zerebralparese. Operat Orthop Traumatol 12:24-39

4. Graf R, Roth-Schiffl E (2001) Offene Reposition der sogenannten kongenitalen Hüftluxation. Operat Orthop Traumatol 13:39-49

5. Weber M, Ganz R (2002) Die Berner periazetabuläre Osteotomie. Operat Orthop Traumatol 14:93112

6. Stark A, Wallenstein R (2003) Periazetabuläre Osteotomie zur Behandlung der Hüftgelenksdysplasie unter Verwendung eines ilioinguinalen Zugangs. Operat Orthop Traumatol 15:269-287

7. Slongo TF (2008) Osteotomien am proximalen Femur bei Kindern. Operat Orthop Traumatol 20:334-353 